\title{
Multimodal structure of baroclinic tides in the South China Sea
}

\author{
V. Vlasenko ${ }^{1}$, N. Stashchuk ${ }^{1}$, C. Guo ${ }^{2}$, and X. Chen ${ }^{2}$ \\ ${ }^{1}$ School of Marine Science and Engineering, Plymouth University, Drake Circus, Plymouth PL4 8AA, UK \\ ${ }^{2}$ Ocean University of China, 238 Songling Road, Qingdao, 266100, China
}

Received: 29 April 2010 - Revised: 26 July 2010 - Accepted: 13 September 2010 - Published: 7 October 2010

\begin{abstract}
The modelling of baroclinic tides generated in the northern South China Sea is studied using a fully-nonlinear non-hydrostatic numerical model. The focus of the modelling efforts was on the vertical structure of internal waves in the vicinity of the Luzon Strait.

The barotropic tidal flow interacting with a two-ridge bottom topography in the area of the Luzon Strait produces a complex baroclinic tidal signal. A multimodal baroclinic bore with counter-phase displacement of isopycnals generated over the ridges and propagating westward disintegrates into a series of large-amplitude solitary internal waves. The leading first-mode solitary wave of depression is followed by a second mode solitary wave coupled with a packet of shortscale internal waves riding it. Scrutiny of the characteristics of the both wave forms, i.e. the carrier second-mode solitary wave and the packet of short waves, revealed that the shortscale waves are basically concentrated in the upper $500 \mathrm{~m}$ layer and attenuate exponentially below it. The short waves exist only thanks to a specific structure of horizontal velocity produced by the second-mode solitary wave. Having equal phase speeds and propagating together for a long distance, this coupled system produces quite a remarkable signal at the free surface, which can be detected by means of remote sensing technique.

It was found in a series of sensitivity experiments that the eastern ridge is responsible for the generation of progressive first-mode tidal waves disintegrated into packets of first-mode ISWs. The western ridge produces quite a strong higher-mode signal. The waves generated over the eastern and western ridges interfere in the near-field, and their nonlinear superposition enhances the multimodal signal in the whole domain.
\end{abstract}

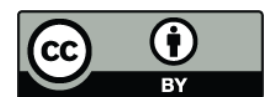

Correspondence to: V. Vlasenko (vvlasenko@plymouth.ac.uk)

\section{Introduction}

Internal solitary waves (ISW) in the northern South China Sea are among the largest ISWs recorded in the World Ocean. They are frequently observed in synthetic aperture radar (SAR) images (Ebbesmeyer et al., 1991; Zhao et al., 2004; Hsu et al., 2000; Du et al., 2008) and in situ data (Ramp et al., 2004; Duda et al., 2004). Statistical analysis of SAR images collected from 1995 to 2001 (Zheng et al., 2007) have shown that almost all ISW packets are concentrated within a latitudinal band from $20^{\circ}$ to $22^{\circ} \mathrm{N}$ and reveal a westward propagation (Fig. 1). It was found that $22 \%$ of these ISW packets are located between $118^{\circ} \mathrm{E}$ and the west of the Luzon Strait (LS). The rest of the wave packets were recorded between $116^{\circ}$ and $118^{\circ} \mathrm{E}$, which is a transition zone between the deep ocean and the continental shelf and where the disintegration and wave breaking takes place (Ebbesmeyer et al., 1991; Zhao et al., 2004; Hsu et al., 2000; Du et al., 2008).

The Luzon Strait with the Batan Islands, Babuyan Islands and submarine ridges lies between Taiwan and the Philippines and is believed to be the generation site of these waves due to a large tidal activity here. Zhao et al. (2004) compiled a spatial distribution map of solitary wave packets recorded during six years (from 1995 to 2001) by overlapping their signatures. In this figure ISWs propagating northwestward are marked in a number of locations in a relatively narrow band elongated from the LS to the shelf of the northern South China Sea, which demonstrates that the ridges in the LS are the most probable site of their generation. According to this research, two types of solitary waves have been identified between $118^{\circ} \mathrm{E}$ and the west of the LS: multiple internal wave packets, and single ISW without tail, Fig. 1. The latter have a crest length of about $150 \mathrm{~km}$ and a width across the wave of about $2.5 \mathrm{~km}$, whereas the former are much shorter, only $0.8 \mathrm{~km}$ wide. The single solitary wave normally appears more than $200 \mathrm{~km}$ away from the ridge, while the packets of shorter internal waves are usually observed in the vicinity of

Published by Copernicus Publications on behalf of the European Geosciences Union and the American Geophysical Union. 


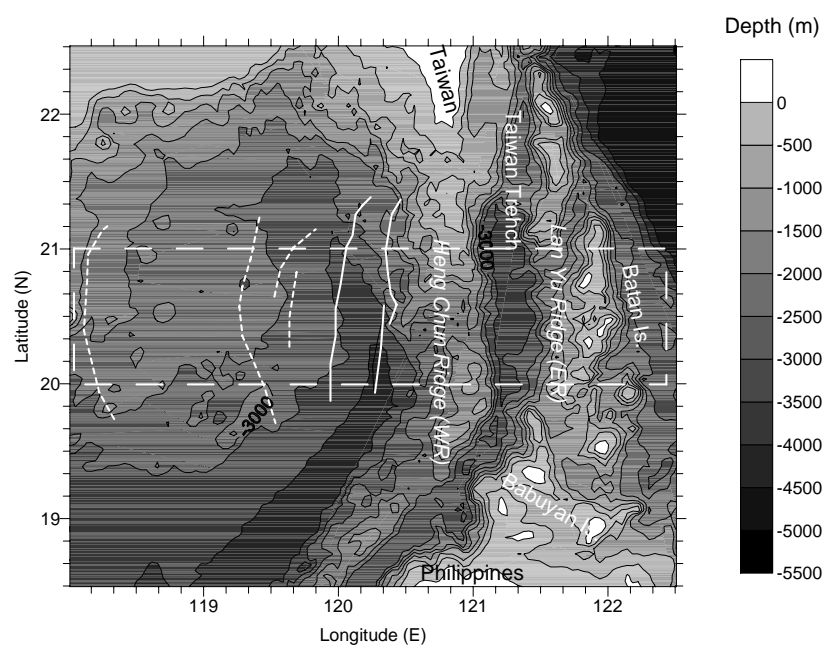

Fig. 1. Model domain showing bottom topography in the Luzon Strait with schematic presentation of generated multiple wave packets (solid lines) and single solitary waves (dotted lines) (Zhao et al., 2004).

the topographic features. Analysis of a series of the satellite images taken from 1995 to 2005 presented by Du et al. (2008) once again shows the evidence of two types of wave packets generated in the Luzon Strait: single "ordinary" ISWs and groups of short waves.

In-situ measurements of ISWs in the northern South China Sea were conducted during a large-scale field experiment ASIAEX (Asian Seas International Acoustic Experiment) in spring 2001. The area of these observations covered the shelf break area bounded by the latitudes from $21^{\circ}$ to $22.5^{\circ} \mathrm{N}$ and longitudes from $117^{\circ}$ to $119^{\circ} \mathrm{E}$. The results of ASIAEX are discussed in a number of papers, i.e. Ramp et al. (2004); Duda et al. (2004); Yang et al. (2004); Zhao and Alford (2006). The remote location of the ASIAEX far from the Luzon Strait does not help in understanding of the origin of two types of internal waves generated in the Luzon Strait.

Some numerical investigations of the generation mechanism of ISWs in the Luzon Strait have been undertaken recently by Du et al. (2008); Warn-Varnas et al. (2010); Buijsman et al. (2010). They include a two-dimensional (2-D) analysis of the baroclinic wave fields in which an idealized bottom topography was used to initiate the generation mechanism. The main outcome from these modelling attempts is that the tidal energy conversion occurred over the doublehead ridge results in formation of a thermocline depression which further transforms into a packet of short waves. However, taking into account a complex three-dimensional bottom bathymetry of the Luzon Strait, it looks unrealistic that 2-D models are able to reproduce all spatial details of the baroclinic wave field because of the wave refraction, interference, diffraction, and unequal conditions for tidal energy conversion at different sites of the strait. In addition, 2-D model experiments failed to to explain the reason for the gen- eration of the two types of waves discussed above. Threedimensional models have recently been applied to the area by Jan et al. (2007) and Shaw et al. (2009), however many details of the generated ISWs were missing because of a number of simplifications and assumptions taken in modelling (hydrostatic assumption for pressure, idealized bottom topography, coarse resolution, etc.).

The Massachusetts Institute of Technology general circulation model (MITgcm) (Marshall et al., 1997) is used in the present study in order to investigate the tidal energy conversion in the Luzon Strait. The aim of this study is to address the problem of finding of the major factors controlling the generation of different types of internal waves. The paper is arranged as follows: the model initialization is described in Sect. 2; Sect. 3 contains the results of the reference experiment, whereas Sect. 4 focuses on the role of the western and eastern ridges in the generation mechanism; the sensitivity of the model results to various input parameters is presented in Sect. 5; the conclusions are formulated in Sect. 6.

\section{Model description and initialization}

The bottom topography in the Luzon Strait and surrounding areas is shown in Fig. 1. Two major bottom features dominate in the strait: the eastern Lan-Yu Ridge and the western Heng-Chun Ridge separated by the Taiwan-Luzon Trench. The eastern ridge is much higher in the middle of the strait while the western ridge is higher at its northern periphery where it connects to the continental shelf. There are several islands (Batan Islands and Babuyan Islands) and underwater banks along the eastern ridge. They are located in the middle and southern part of the strait.

Not all fragments of the strait bathymetry participate equally in the generation processes. According to Zheng et al. (2007) the waves appear in a narrow band between latitudes $20^{\circ}$ and $21^{\circ} \mathrm{N}$ (see Fig. 1). Therefore the model domain with real bottom topography was restricted by these two boundaries (find dashed rectangle in Fig. 1). The model uses a Cartesian system of coordinates $(0 x y z)$, with $0 x y$-plane parallel to the undisturbed free surface and the $0 z$-axis directed vertically upward, and the $0 x$ - and $0 y$-axes directed eastward and northward, respectively.

The size of the model domain is $L_{x} \times L_{y}=670 \times 134 \mathrm{~km}^{2}$. It includes a rectangular grid with the resolution $\Delta x=$ $250 \mathrm{~m}, \Delta y=1000 \mathrm{~m}$. Four sponge areas were added to the lateral "fluid" boundary of the domain with an increase of the resolution from $\Delta x=250 \mathrm{~m}$ to $10^{7} \mathrm{~m}$ in the $\mathrm{x}$-direction and from $\Delta y=1000 \mathrm{~m}$ to $10^{5} \mathrm{~m}$ in the $\mathrm{y}$-direction. Such a procedure allows one to avoid reflection of both barotropic and baroclinic waves from the lateral boundaries. In the vertical direction $90 \mathrm{z}$-levels were used with $\Delta z=10 \mathrm{~m}$ resolution in upper $500 \mathrm{~m}$ layer (main thermocline) followed by 20 intermediate layers with $\Delta z=50 \mathrm{~m}$ resolution, and finally the bottom 20 layers with $\Delta z=150 \mathrm{~m}$. The initially 
homogeneous domain-wide distribution of temperature $T(z)$, and salinity, $S(z)$, are taken from World Ocean Atlas (2005), Fig. 2.

The model is forced by a periodical tidal flow propagating through the strait in an east-west direction. The tidal dynamics in the area of the northern South China Sea is governed by a superposition of eight principal tidal harmonics, $\mathrm{M}_{2}, \mathrm{~S}_{2}$, $\mathrm{K}_{1}, \mathrm{O}_{1}, \mathrm{~N}_{2}, \mathrm{~K}_{2}, \mathrm{P}_{1}, \mathrm{Q}_{1}$ (Egbert and Erofeeva, 2002). Note, however, that according to $\mathrm{Zu}$ et al. (2008) not all of them are equally important for the tidal energy conversion. The comparison of the model output conducted for only $\mathrm{M}_{2}$ and $\mathrm{K}_{1}$ with a similar solution obtained for eight harmonics has shown that there is little difference between them. This is the reason why only $\mathrm{M}_{2}$ and $\mathrm{K}_{1}$ constituents are included in our analysis.

Another key issue concerns the efficiency of the baroclinic tide generation under the action of the Earth's rotation. The tidal energy conversion near critical latitudes is inhibited by the rotation. The Coriolis force produces along-front currents which are partly in geostrophic balance with the crosswave density gradients. These quasi-geostrophic currents, in turn, suppress the wave steepening and its disintegration into solitary waves (Gerkema, 1996; Helfrich and Grimshaw, 2008; Stashchuk and Vlasenko, 2005). The model domain is located between the longitudes $20^{\circ}$ and $21^{\circ} \mathrm{N}$ (the latitude $30^{\circ} \mathrm{N}$ is critical for the $\mathrm{K}_{1}$ diurnal harmonic, and the latitude $74^{\circ} 30^{\prime} \mathrm{N}$ is critical for the semi-diurnal $\mathrm{M}_{2}$ harmonic). This means that an efficient tidal energy conversion and nonlinear evolution at $\mathrm{K}_{1}$ frequency is expected to be weak in comparison with $\mathrm{M}_{2}$ baroclinic tidal response. While the diurnal baroclinic activity is suppressed by the rotation, the semidiurnal tide can effectively generate internal waves in the northern South China Sea (Helfrich and Grimshaw, 2008). To confirm the prevailing role of semi-diurnal tides in the generation process, Zhao and Alford (2006) showed a strong correlation between internal wave occurrences near Dongsha coral reef $\left(117^{\circ} \mathrm{E}, \mathrm{ASIAEX}\right)$ and tidal current at the Luzon Strait as dominantly semi-diurnal in character. All above reasonings justify our approach to consider $\mathrm{M}_{2}$ and $\mathrm{K}_{1}$ harmonics separately (see section "Sensitivity runs" for more details).

The model is forced from the state of rest by a periodical barotropic tidal flow with tidal frequency $\sigma$ and amplitude of horizontal velocity $U$. In doing so the following components of an external force, $F_{x}=U H_{0} / H(x, y) \sigma \cos (\sigma t)$ and $F_{y}=U H_{0} / H(x, y) f \sin (\sigma t)$, are added to the zonal and meridional momentum balance equations. Here $H_{0}$ is the depth where the tide has velocity $U, H(x, y)$ is the basin depth, and $f$ is the Coriolis parameter.

It is expected that the barotropic tidal flow propagating over a complex terrain with islands and steep bottom features generates considerable water mixing. To keep the model running the Richardson number-dependent parameterizations (Pacanowski and Philander, 1981) were used for calculations of vertical viscosity $v$ and diffusivity $\kappa$ :

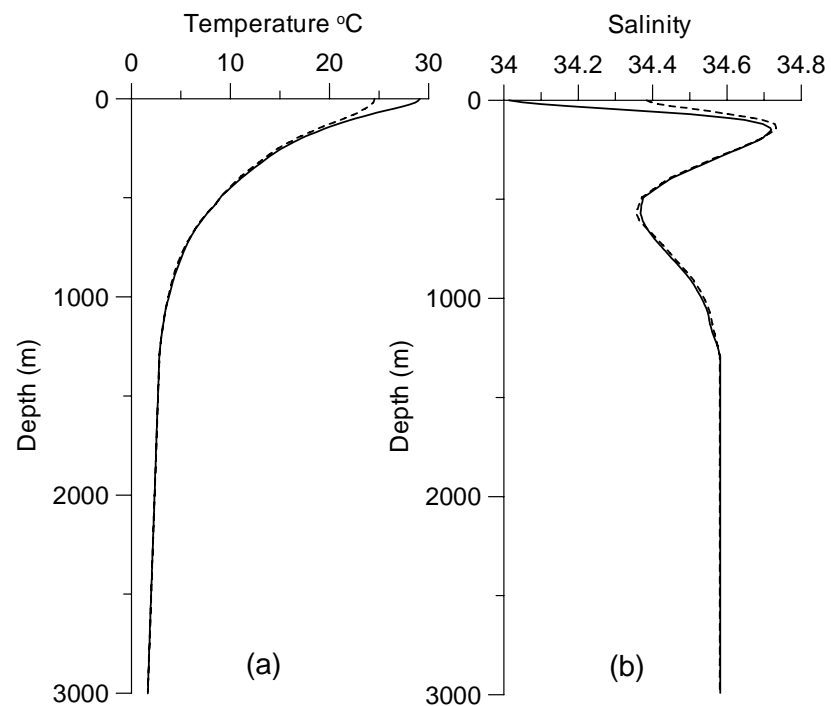

Fig. 2. Averaged temperature (a) and salinity (b) profiles for summer (solid lines) and winter (dashed lines) seasons used in modelling.

$v=\frac{v_{0}}{(1+\alpha R i)^{n}}+v_{b}, \quad \kappa=\frac{v}{(1+\alpha R i)}+\kappa_{b}$,

Here $R i$ is the Richardson number, $R i=N^{2}(z) /\left(u_{z}^{2}+v_{z}^{2}\right)$, $N(z)$ is the buoyancy frequency, $u$ and $v$ are the horizontal velocity in $\mathrm{x}$ - and $\mathrm{y}$-directions; $v_{b}=10^{-5} \mathrm{~m}^{2} \mathrm{~s}^{-1}$ and $\kappa_{b}=10^{-5} \mathrm{~m}^{2} \mathrm{~s}^{-1}$ are background parameters, $\nu_{0}=1.5 \times$ $10^{-2} \mathrm{~m}^{2} \mathrm{~s}^{-1}$, and $\alpha=5$ and $n=1$ are adjustable parameters. Such a parameterization increases $v$ and $\kappa$ in areas where the Richardson number is small. The horizontal viscosity was calculated using the scheme due to Leith (Leith, 1996).

\section{Reference experiment}

We start our analysis with the reference experiment conducted for summer stratification (Fig. 2) when the semidiurnal tide with maximum horizonatal velocity $U=$ $0.13 \mathrm{~m} \mathrm{~s}^{-1}$ at depth of $3000 \mathrm{~m}$ is used as a forcing (this value is typically predicted by TPXO7.1 model, Egbert and Erofeeva, 2002). The surface signature of generated internal waves is shown in Fig. 3 after five tidal cycles. As it can be inferred from Fig. 3, the horizontal structure of generated waves in the near-field of the Luzon Strait (more specifically, to the east from $120^{\circ} \mathrm{E}$ ) is substantially three-dimensional. At the same time to the west from $120^{\circ} \mathrm{E}$ the surface signature of internal waves is basically two-dimensional with wave fronts elongated in south-north direction, although the variability of a signal along the wave fronts is also evident. The vertical structure of temperature fields taken in sections $a, b, c, d$ shown by dashed lines in Fig. 3 can illustrate spatial variability of the generated wave fields. Four temperature sections are presented in Fig. 4 (the original labeling of sections in Fig. 3 coincides with names of panels in Fig. 4). 


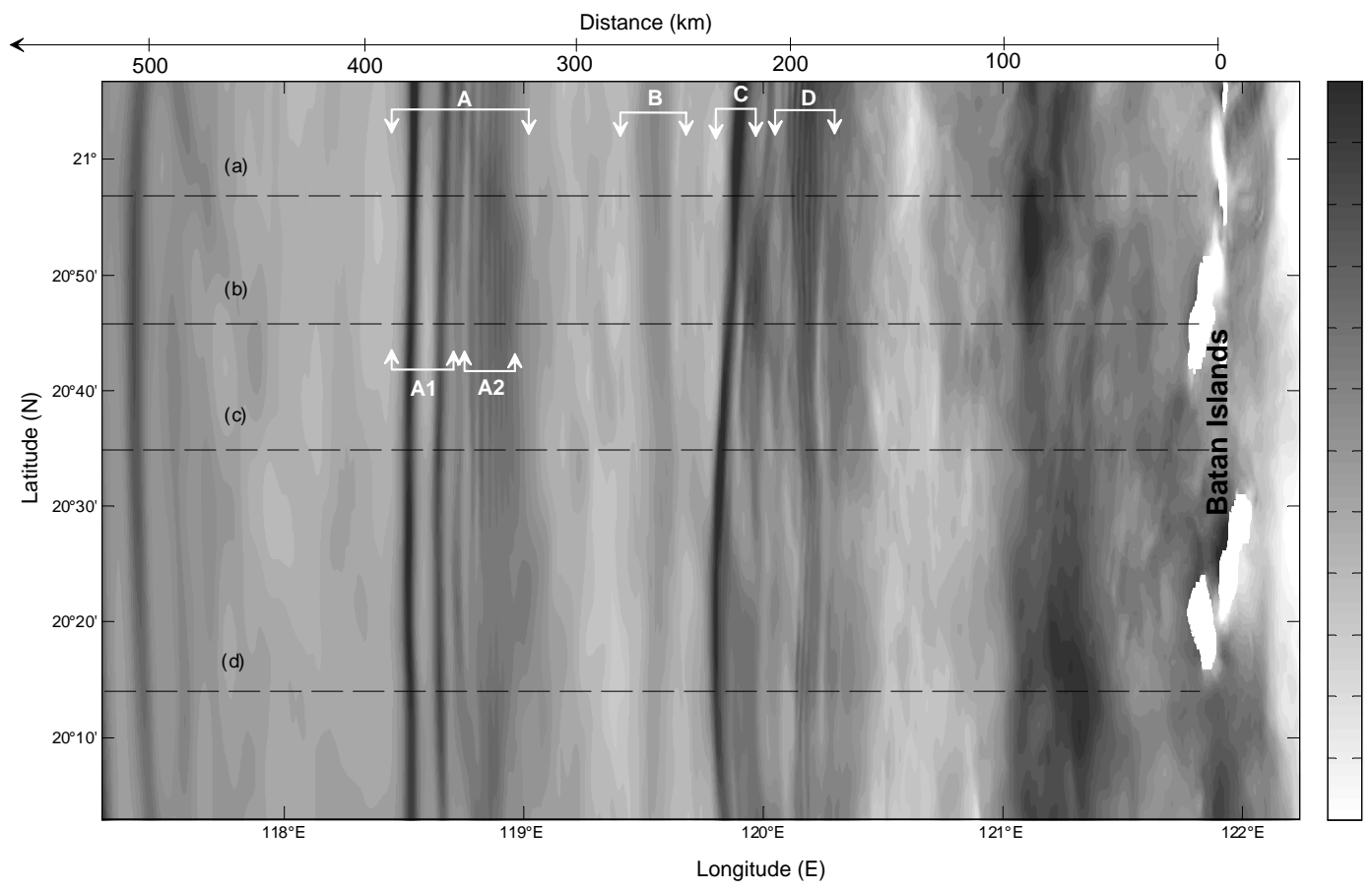

Fig. 3. Model-predicted sea surface elevations produced by internal waves generated at the Luzon Strait and propagated westward. The axis at the top shows an actual coordinate system used in Figs. 4, 7 and 8. Wave structures marked as $C$ and $D$ correspond to the similar fragments II and III shown in Fig. 7h.

Analysis of the sections presented in Fig. 4 shows that all of them contain several similar wave fragments; they are shown by shaded areas and marked by letters $A, B, C, D$. It seems that the fragment $A$ represents a developing packet of solitary waves of depression in the frontal side and a secondmode-type solitary wave at its trail edge. Vertical excursion of isotherm in the centre of the leading first-mode wave exceeds $100 \mathrm{~m}$, and the amplitude of the second-mode-type wave is about $80 \mathrm{~m}$. It should be noted that the wave element $A$ is not completely identical in all four sections because of the spatial variability.

Figure 5a represents a close-up of two leading solitary waves of depression located in the fragment $A 1$; corresponding horizontal velocity field is shown in Fig. 5b. Qualitative analysis of Fig. 5 allows one to classify both waves as the first-mode solitary waves of depression. Their maximum vertical excursion is located just below the density jump. The horizontal velocity presents current-countercurrent distribution typical for traditionally observed oceanic solitary waves. Although both waves are still not well separated in space, they definitely can be identified as first-mode solitary waves of depression.

The counterphase displacements of isotherms in the subfragment $A_{2}$ shown in Fig. $4 \mathrm{~b}$ (depressions above the thermocline and elevations below it) allow us to assume that its structure is close to a second-mode solitary wave. Figure 6 represents vertical profiles of the isopycnal displacements (panel a), horizontal (panel b) and vertical (panel c) velocities of the second-mode wave taken in its centre (dashed line in the middle of the fragment $A_{2}$, Fig. $4 \mathrm{~b}$ ). Similarly three profiles calculated by solving a standard eigen-value problem for an 80-m amplitude second-mode internal wave are shown by dashed lines in Fig. 6. The coincidence of the numerical and theoretical curves in Fig. 6 is good enough to conclude that the sub-fragment $A_{2}$ may be treated as a second mode wave. Thus the fragment $A$ (Figs. 3 and 4) contains a packet of first mode solitary waves followed by a second mode wave.

The fragment $B$ in Fig. 4 contains only one solitary wave which has a structure of second baroclinic mode with counterphase displacement of isopicnals above and below the depth of $300 \mathrm{~m}$. This wave is traced through the whole area from south to north, although at the southern periphery (panel d) it is not as pronounced as at the northern periphery (panel a).

The last two fragments in Fig. 4 show the well developed solitary wave of depression, fragment $C$, and well developed second-mode irregular waves covered by the fragment $D$. The spatial south-north variability of these waves is quite prominent, although these waves basically preserve their form in the whole area.

Thus, the analysis of Fig. 4 has shown that after five tidal cycles the generated wave field in the area of the LS is substantially three-dimensional with clear evidence of multimodal structures in which the second-mode waves are 

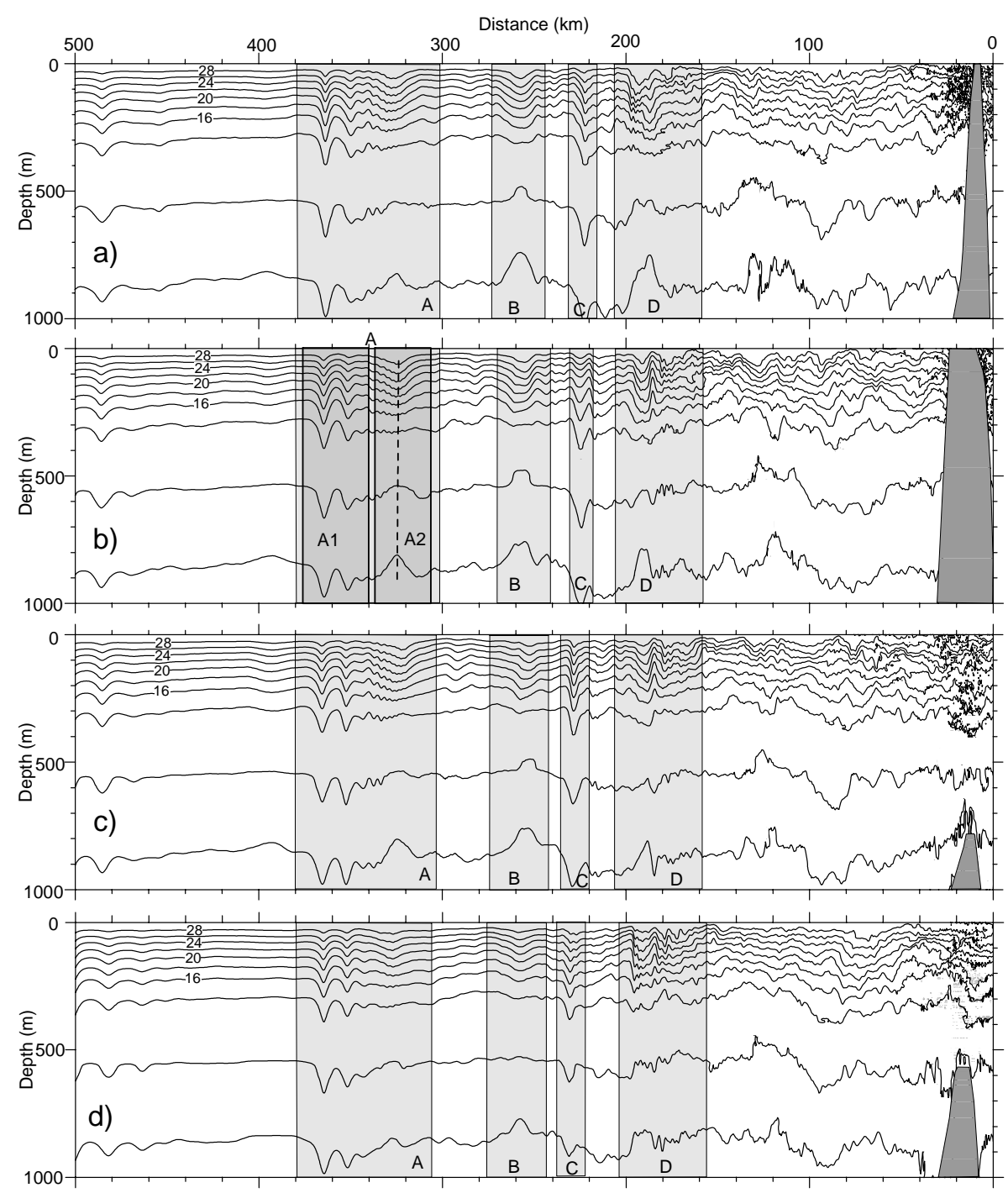

Fig. 4. Model predicted temperature fields after five tidal periods. Panels (a-d) correspond to similar cross-sections shown in Fig. 3. Fragments $A, B, C$ and $D$ represent similar wave fragments but at different locations.

comparable in amplitude with their first-mode counterparts. In addition, the second mode in fragments $A$ and $D$ are accompanied by a series of short periodical internal waves which produce fairly strong signals at the free surface (find fragment $A_{2}$ and $D$ in Fig. 3). The generation mechanism and the spatial/temporal characteristics of these short waves are discussed below in detail.

To understand the origin of the short waves let us consider the evolution of the fragment I marked in Fig. 7 by shaded area. In the course of the evolution the frontal edge of fragment I gradually transforms into a baroclinic bore with gently sloping leading and steep rear faces, Fig. 7a. Note, however, that this baroclinic bore reveals vertical counter-phase displacements of isotherms above and below $300 \mathrm{~m}$, which points out the presence of higher modes. In other words, the baroclinic energy in the wave fragment I is concentrated not only in the lower, but also in higher modes.
It is important to note that a propagating first-mode baroclinic bore usually disintegrates into a packet of well rankordered first-mode solitary waves. It seems that Fig. 7a, b and $\mathrm{c}$ represent an initial stage of such a process. However, the next time span presented in Fig. 7d reveals that the baroclinic bore evolves into a number of wave forms that are not the first mode objects. The most probable reason for the generation of higher-mode waves in such a case is the multimodal structure of the initial bore, Fig. 7a. As a result, in the course of its disintegration the energy is partly transformed to higher-mode waves.

It is clear that higher modes propagate slower than the first one, and the modes separate in space and time, as it is seen in Fig. 7e and f. The leading solitary wave in the fragment I (Fig. 7e) is a first-mode solitary wave of depression. This wave moving with the larger phase speed increases the distance to the rest of the wave field (find fragment II in Fig. 7h), 


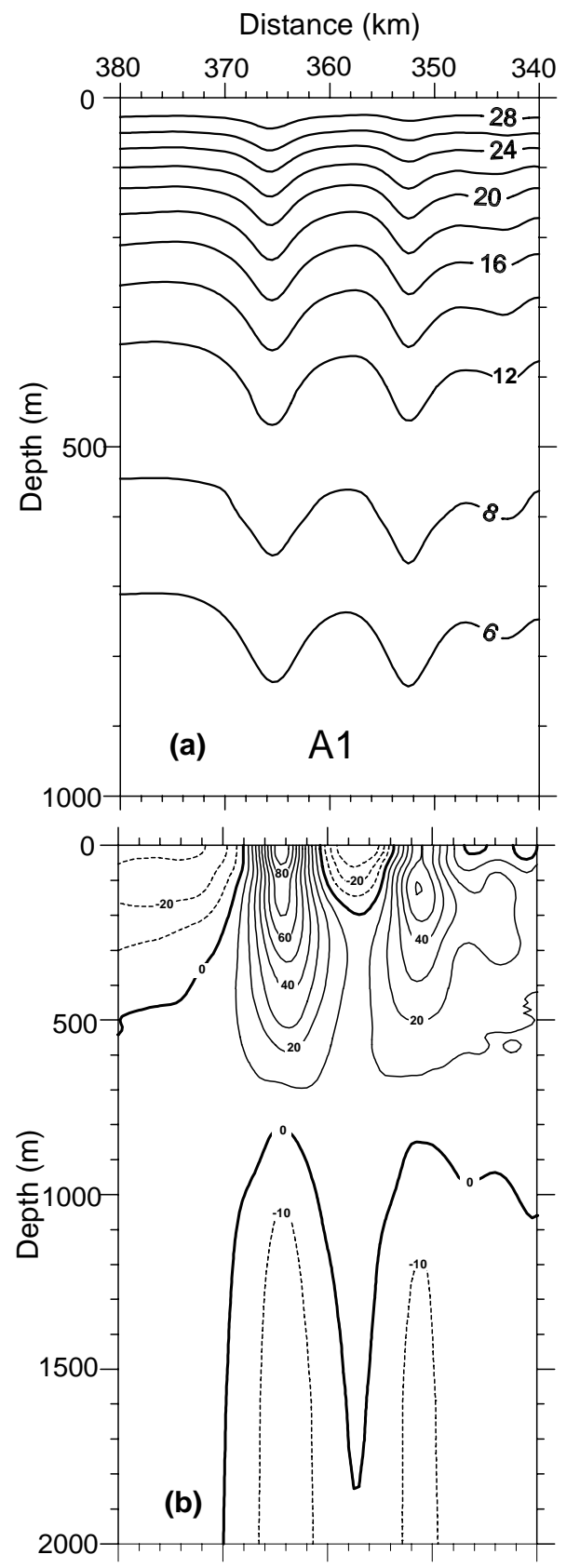

Fig. 5. A pair of first-mode solitary waves located in the rectangle $A 1$ shown in Fig. 4b. Panel (a) shows the temperature field $\left({ }^{\circ} \mathrm{C}\right.$ ) and panel (b) depicts the horizontal velocity field $\left(\mathrm{cm} \mathrm{s}^{-1}\right)$.

and by the end of the tidal cycle it completely separates from the tail.

The remaining fragment III represents a second mode solitary wave with the structure similar to those shown in Fig. 6. Note that both second-mode ISWs, the fragment $A_{2}$ shown in Fig. $4 \mathrm{~b}$ and the fragment III shown in Fig. $7 \mathrm{~g}$, contain packets of short quasi-periodic waves with wavelength of about $1.5 \mathrm{~km}$ and amplitude of $20 \mathrm{~m}$ riding the main second-
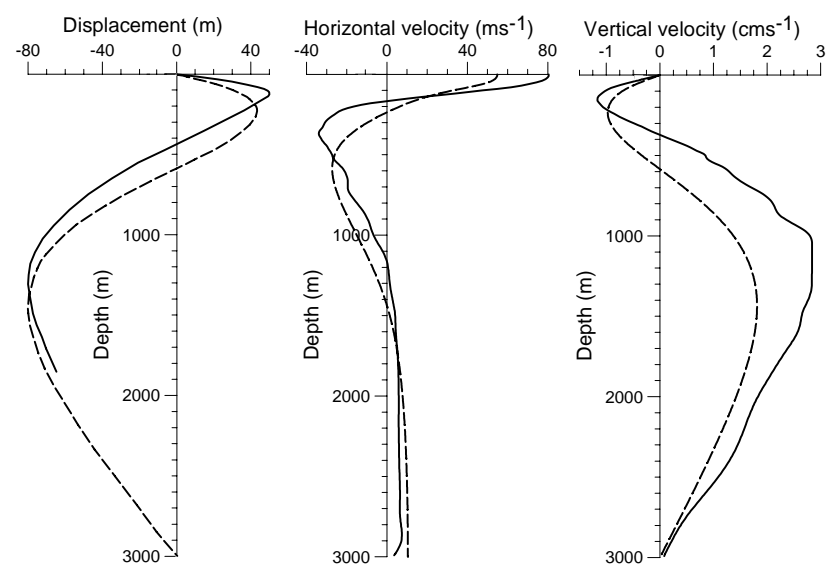

Fig. 6. Vertical profiles of a second-mode solitary wave in its centre (fragment $A_{2}$, Fig. 4b) as predicted by numerical model (solid lines) and calculated from a standard boundary-value problem (dashed lines).

mode solitary wave. A close-up of the fragment III, Fig. 8c, and surface elevation, Fig. 8a, show these short waves quite clearly. It is seen that these waves are well developed in the upper $500 \mathrm{~m}$ layer but almost invisible below it. The parameters of the short-scale waves, i.e. their structure function $g(z)$ presenting vertical isopycnal excursion and phase speed $c$ can be discovered solving the following eigen-value problem:

$\frac{d^{2} g_{j}}{d z^{2}}+\left\{\frac{N^{2}(z)}{[U(z)-c]^{2}}-\frac{U^{\prime \prime}(z)}{(U(z)-c)}-k^{2}\right\} g_{j}=0$,

$g_{j}(-H)=g_{j}(0)=0, \quad j=1,2,3, \ldots$

Here $N(z)$ is the buoyancy frequency, $U(z)$ is the vertical profile of the background current $\left(U^{\prime \prime}(z)\right.$ is its second $z$ derivative), $H$ is the water depth, and $k$ is the wavenumber. This eigen-value problem (Eqs. 1-2) has a set of eigen functions $g_{j}(z)$ with appropriate eigen-values $c_{j}$. For oscillating internal waves the term in brackets must be positive, otherwise the character of the solution is exponential. Transition from the layers with positive to negative term leads to change of the character of the solution from oscillatory to exponential.

Looking at Fig. 8c one can conclude that the amplitude of short waves in vertical sections $a-a, b-b$ and $c-c$ are well developed above $500 \mathrm{~m}$, and almost invisible below this depth. In order to check whether the second-mode baroclinic ISW with a specific shear-current profile $U(z)$ can generate such waves, the Taylor-Goldstein Eq. (1) with boundary conditions (2) was solved. Taking into account that the short waves are well preserved during a long period of time riding the second mode solitary wave, it is clear that their phase speed and spatial structure (both vertical and horizontal) are consistent with the stratification and horizontal shear 


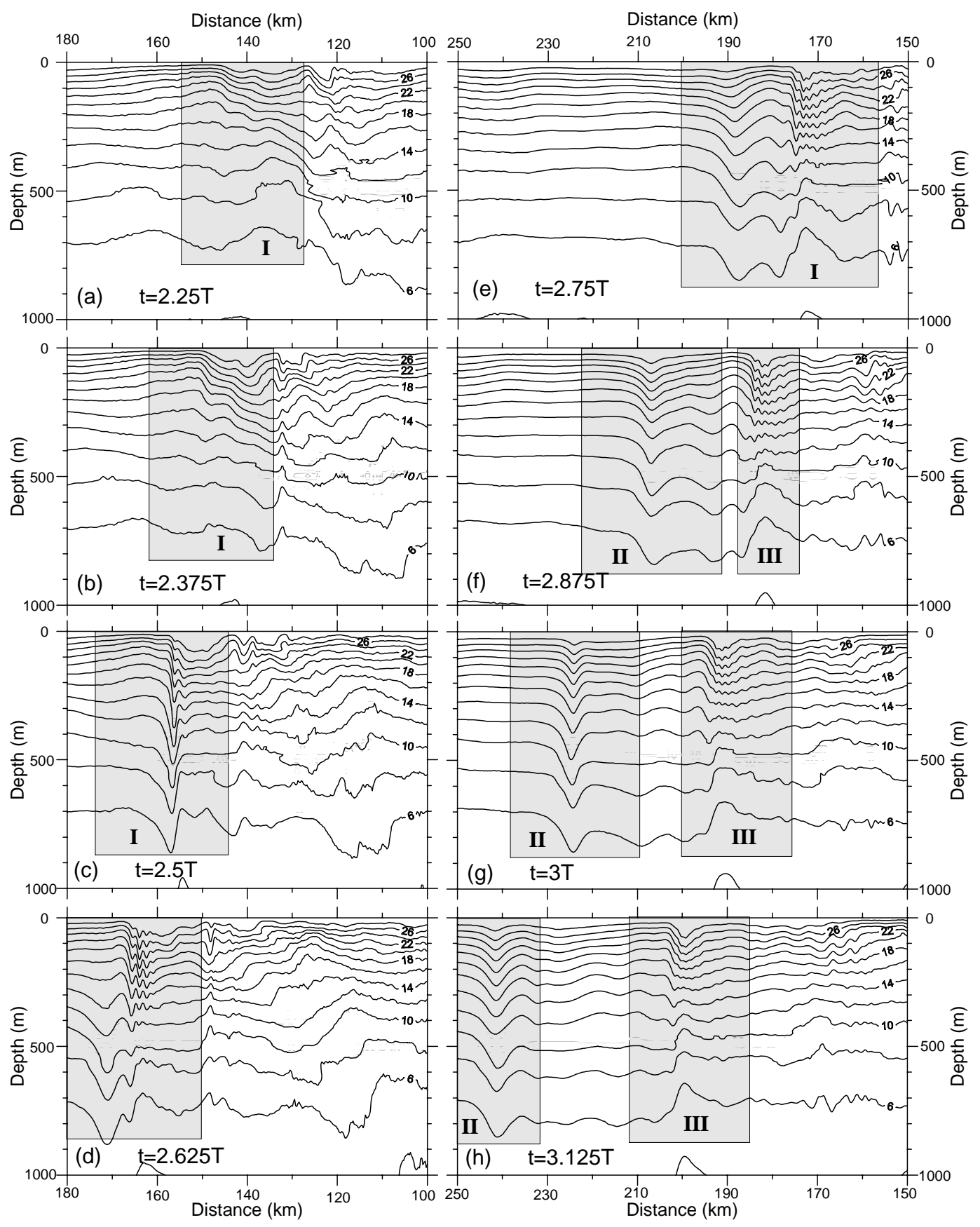

Fig. 7. Evolution of a multimodal baroclinic bore during one tidal cycle taken along the section (b), see Fig. 3.

currents induced by a second-mode wave. If so, the input buoyancy frequency profile $N(z)$ (or density $\rho(z)$ ) as well as the velocity profile $U(z)$ for eigen-value problem (1-2) should be taken in the middle of the fragment III presented in Fig. 7. Such profiles for the three moments of time, i.e. for $t=2.875 T, 3 T$ and 3.125T (see panels f, g, and $\mathrm{h}$ in Fig. 7) are shown in Fig. 9. Fairly good coincidence of the density profiles suggests that the vertical stratification generated by the carrier second-mode waveform is fairly stable at differ- ent phases of the tidal cycle, whereas presented $U$-profiles are modified by the barotropic tidal current.

Three sets of input conditions shown in Fig. 9 are used to find the eigen-functions and eigen-values of the problem (1$2)$. The wavelength $\lambda=1.5 \mathrm{~km}$ of the short waves was estimated from Fig. 8. The vertical structure of the first eigenfunction $g_{1}(z)$ is shown in Fig. 10 for three moments of time, $t=2.875 T, 3 T$ and $3.125 T$. 
(a)

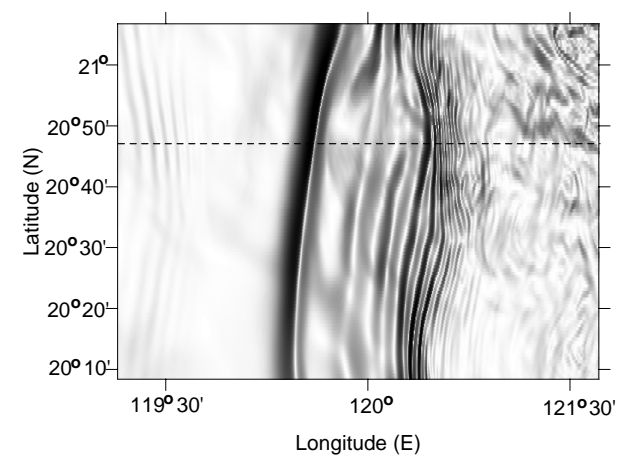

(b)

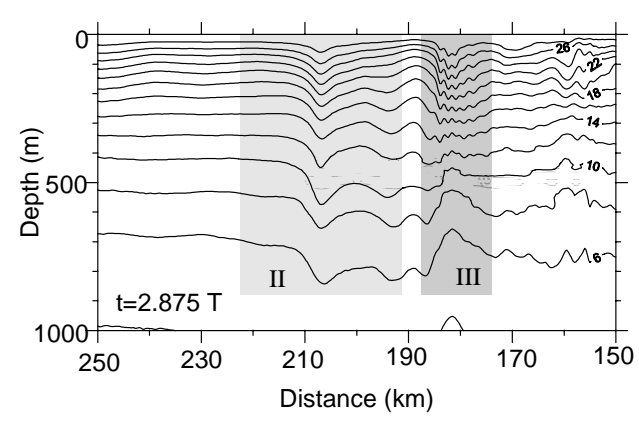

(c) Distance $(\mathrm{km})$

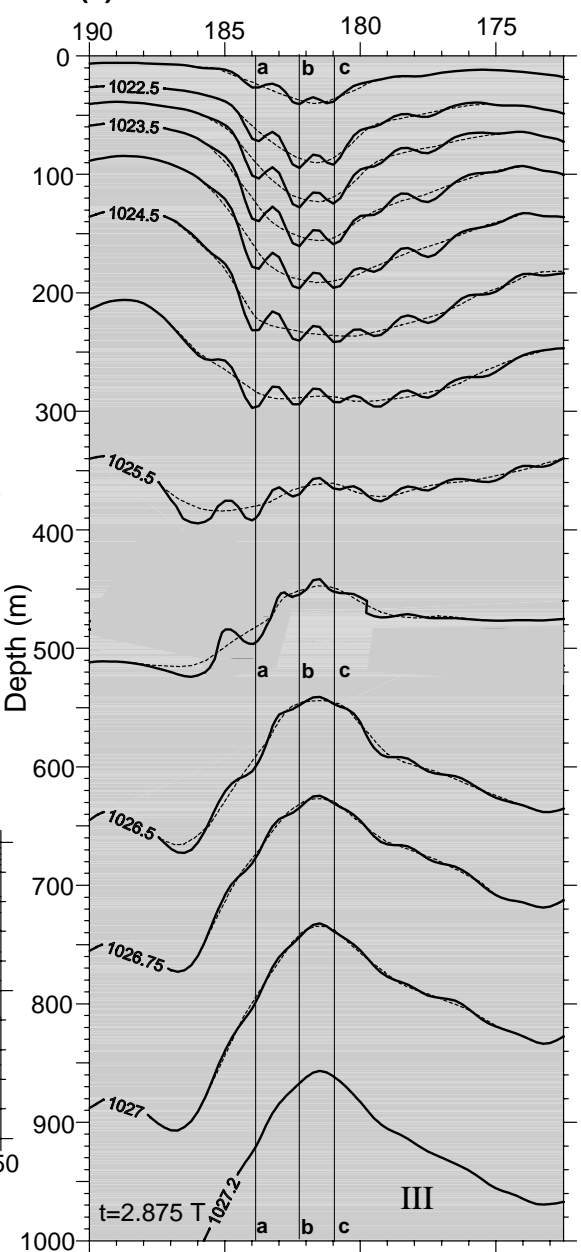

Fig. 8. (a) Surface signature of first-mode solitary waves and packet of short waves at $t=2.875 T$. (b) Model-predicted temperature field along the cross-section shown in panel (a) by a dashed line. (c) Zoom of the element III as a density field. Dashed lines represent averaged isopicnals of a 2-nd mode solitary wave after removal of short-scale oscillations.
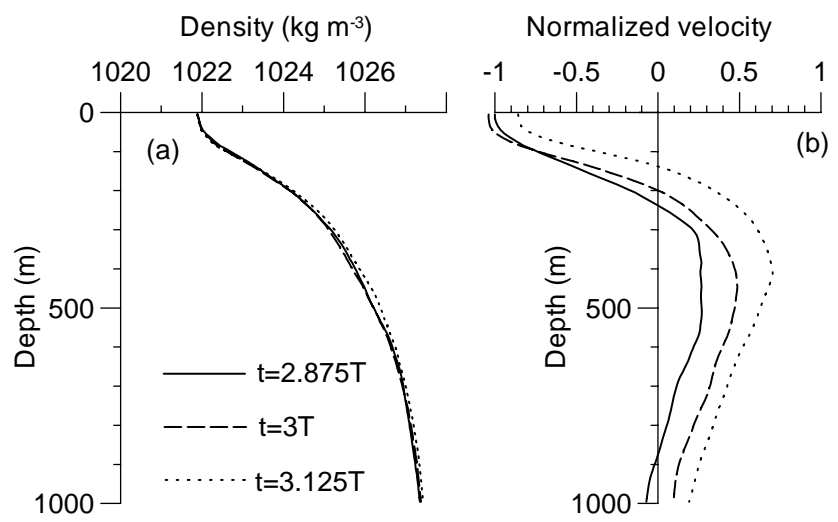

Fig. 9. Vertical profiles of density and normalized horizontal velocity taken in the middle sections of the wave fragments III shown in Fig. 7f, $\mathrm{g}$ and $\mathrm{h}$ at $t=2.875 T, 3 T$ and $t=3.125 T$.
As it is seen from the graphs, three eigen-functions reveal exponential decay of the solution below $500 \mathrm{~m}$ and basically coincide with small discrepancy in the position of their maximum. The amplitudes of short waves were calculated for three vertical sections shown in Fig. 8 as a deviation of the isopicnals from their averaged position as shown by the dotted lines. Their normalized values for sections $a-a$, $b-b$ and $c-c$ are given in Fig. 10 by triangles, filled circles and crosses, respectively. Figure 10 shows good coincidence between vertical profiles of the short-scale waves obtained in the numerical experiments and calculated from the eigen-value problem (1-2). The phase speed $c_{1}$ was calculated from Eqs. (1-2) for three density profiles shown in Fig. 9. Three values of $c_{1}$ are: $1.84,1.77$ and $1.55 \mathrm{~ms}^{-1}$ for $t=2.875,3$ and 3.125T, respectively. The actual phase speed of the carrier wave calculated directly from Fig. 7 presents the value of $1.65 \mathrm{~ms}^{-1}$. Fairly good coincidence between numerical and theoretical phase speeds is obvious. 


\section{Effect of two-ridge bottom topography}

Analysis of the numerical runs presented in the previous section have shown that two types of wave packets are generated at the Luzon Strait. They have been classified as traditionally observed first-mode ISWs of depression (1-2 waves in a packet), and multiple short-scale waves riding the carrier second-mode solitary waves. Looking at the wave signature produced by these waves at the free surface one can conclude that the fragment $C$ shown in Fig. 3 can be treated as a single ISW without wave tail, and the fragment $D$ is a multiple internal wave packet, as detected by SARs in real remote sensing observations. The positions of the fragments $C$ and $D$ basically coincide with the longitude $120^{\circ} \mathrm{E}$ where similar waves are usually observed (Zhao et al., 2004, their Fig. 2). As it was shown in the previous section, both systems of waves are generated in the course of disintegration of a multimodal baroclinic bore.

Such a modal diversity of baroclinic tidal signal in the LS can be explained in terms of the mechanism of tidetopography interaction. According to linear theory the first baroclinic mode is mostly generated by tidal wave when subcritical conditions for the bottom inclination are realized. In other words, in a sub-critical case the bottom steepness $\gamma=d H / d x$ is much less than the inclination of the characteristic lines $\alpha=\sqrt{\left(\sigma^{2}-f^{2}\right) /\left(N(z)^{2}-\sigma^{2}\right)}$. In the opposite case, i.e. when $\alpha \approx \gamma$ (near-critical case) or $\alpha>\gamma$ (supercritical case), a wide spectrum of baroclinic modes with comparable amplitudes are generated. Being superimposed these modes create a tidal beam.

As it has been mentioned above, two topographic features are responsible for the generation of baroclinic tides in the area of the Luzon Strait: the eastern Lan-Yu Ridge (ER) and western Heng-Chun Ridge (WR). A 3-D projection of the bathymetry presented in Fig. 1 shows that these ridges are quite different in shape and height, which is why they can produce substantially different baroclinic tidal signals (Vlasenko et al., 2005). The ER is much higher than the western one (in some places it becomes islands), and it is expected that the major part of tidal energy conversion takes place just above the eastern ridge. The WR is deeper but steeper, so one can assume that the western ridge is a potential place for generation of higher baroclinic modes.

To understand the role of every ridge in the mechanism of tidal energy conversion and to localize the area of internal wave generation, two extra numerical experiments were conducted. The model was run with two "truncated" ad-hoc bottom topographies: the first experiment was performed without the WR, and the second experiment was conducted without the ER. The temperature cross-sections along the latitude $20^{\circ} 46^{\prime} \mathrm{N}$ (line (b) in Fig. 3) for "full-topography" experiment and two "truncated" profiles are presented in Fig. 11.

Analysis of the obtained wave fields shows that in the experiment when only the ER is present in the model the baroclinic wave field in the far-field is basically a superposition

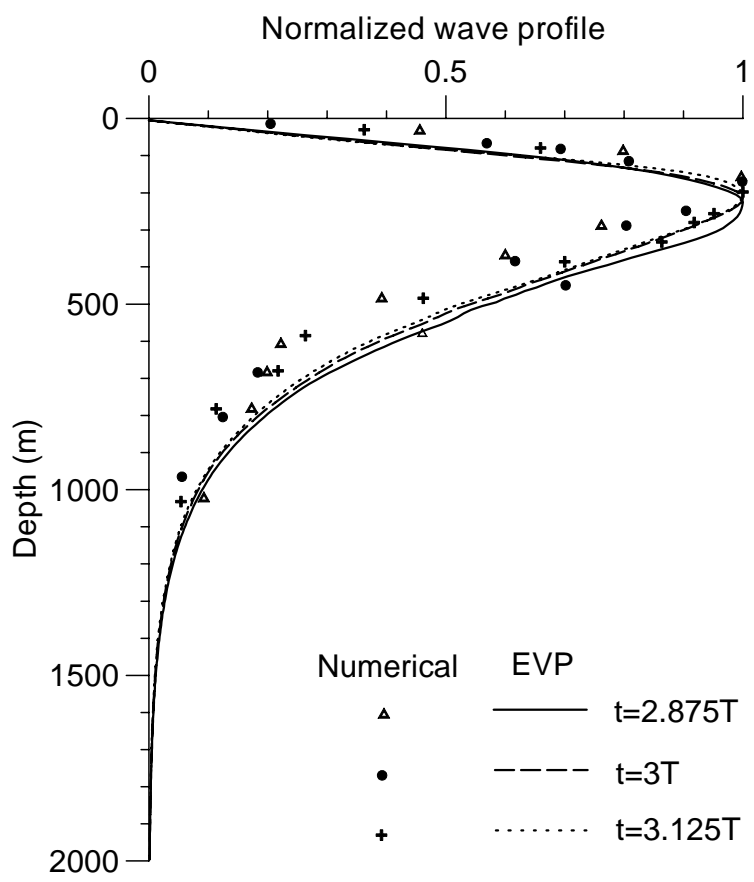

Fig. 10. Normalized profiles of vertical displacement of short waves calculated in vertical sections $a-a, b-b$, and $c-c$ shown in Fig. 8. Vertical displacement was calculated as a distance between solid and dashed lines (see Fig. 8). Solid, dashed and dotted lines represent the vertical profiles of the first eigen function of the eigen-value problem (Eqs. 1-2) calculated for $t=2.875 T, 3.0 T$ and 3.125T, respectively.

of the first-mode baroclinic waves with co-phase vertical displacement of isotherms (Fig. 11b). Only in the close proximity to the ER there is some evidence of higher modes, but they do not propagate far beyond the generation area and do not affect the far-field. If the second-mode waves with remarkable amplitude were radiated from the ridge, after five tidal cycles they would appear at a distance farther than $300 \mathrm{~km}$ from the ridge (wavelength of 2nd-mode wave is of about $75 \mathrm{~km}$ ), but this is not the case. At the same time the propagating first-mode tidal wave effectively disintegrates into a packet of rank-ordered solitary waves (two of them are seen in Fig. 11b) due to nonlinearity and nonhydrostatic dispersion.

A completely different scenario of wave generation and evolution is realized over the WR (see Fig. 11c). There is no strong first-mode baroclinic tidal signal in the far-field. Instead, the structure of isotherms clearly illustrates the generation of two tidal beams emanated from either sides of the ridge. The dashed lines in Fig. 11c show the position of the beams and possible sites of their generation.

Comparing the wave fields calculated for two "truncated" topographies (Fig. 11b and c) with similar output obtained for the real topography (Fig. 11a) one can formulate a conclusion that a joint wave field is not just a linear sum of two 


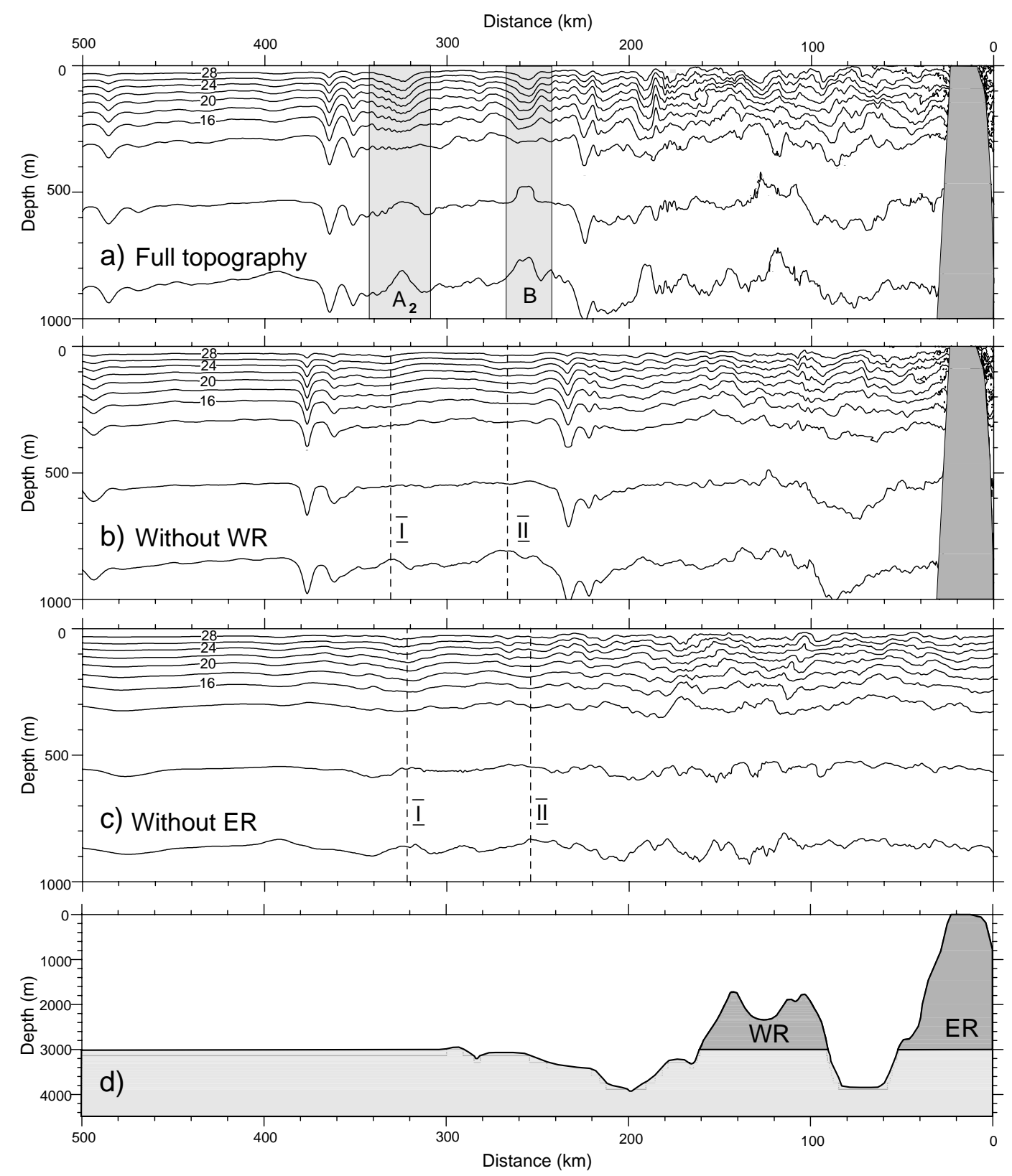

Fig. 11. Model-predicted temperature fields in the cross-section (b), Fig. 3, after five tidal cycles calculated for (a) real topography, (b) without western ridge, and (c) without eastern ridge. The three bottom profiles are shown in panel (d).

baroclinic tidal signals generated over eastern and western ridges, but probably is a result of their nonlinear superposition and mutual amplification. The last remark basically concerns the second-mode signal. Really, the parameters of the first-mode solitary waves in Fig. 11a and b are almost identical. This coincidence testifies that the eastern ridge is responsible for the generation of the first-mode solitary waves. The WR has a weak influence on their characteristics except for probably a little shift caused by the absence of the western ridge in Fig. 11b. As a result, the centre of the leading ISW in the experiment without the WR is located at the distance of $378 \mathrm{~km}$ (Fig. 11b) whereas with full topography the same solitary wave is seen at the distance of $363 \mathrm{~km}$ (Fig. 11a). At the same time the well developed second-mode solitary waves marked by the fragments $A_{2}$ and $B$ in the experiment with full topography (Fig. 11a), are very weak in experiments with truncated topographies (marked as I and II in Fig. 11b and c).

A possible explanation of an efficient second-mode generation over the real topography can be found in terms of a resonant excitement of waves in a two-ridge system and their further nonlinear interference and amplification. The 


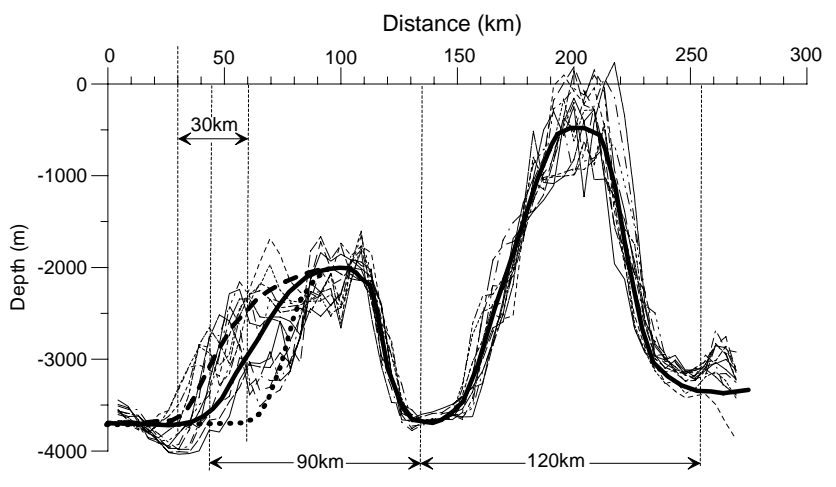

Fig. 12. Several equidistant zonal bottom profiles taken between $20.25^{\circ} \mathrm{N}$ and $20.75^{\circ} \mathrm{N}$ (see Fig. 3) are shown by thin lines of different style. Thick solid line represents an average bottom profile with thick dashed and dotted lines showing the divergence.

conditions for the resonant excitement of internal waves in an underwater ridge of height $H_{m}$ can be studied using analytical solution developed by Vlasenko et al. (2005). Beyond the topographic feature this solution represents a superposition of propagating baroclinic modes. In terms of vertical displacement $\zeta(x, z, t)$ this solution reads:

$\zeta(x, z, t)=\epsilon \sum_{j=1}^{\infty} a_{j} g_{j}(z) \exp \left[i\left(k_{j} x-\sigma t+\varphi_{j}\right)\right]$,

where $a_{j}$ is the amplitude of $j$-th baroclinic mode $(j=1,2,3, \ldots), g_{j}(z)$ and $k_{j}$ are its vertical profile and wavenumber, respectively, defined from a standard eigenvalue problem. In this formula the parameter $\epsilon$ is an "efficient" height of the bottom topography, i.e. $\epsilon=$ $\int_{-H+H_{m}}^{-H} N(z) d z / \int_{0}^{-H} N(z) d z$ Where $H$ is the ocean depth, $H_{m}$ is the ridge height, and $\varphi_{j}$ is the phase of generated $j$-th mode. Exact representation of all parameters and functions can be found in Vlasenko et al. (2005).

The amplitudes of generated waves for the eastern and western ridges, $a_{j}^{e}$ and $a_{j}^{w}$, were calculated separately. The idealized form of the bottom topography was found after averaging of 13 equidistant zonal cross-sections taken from $20.25^{\circ} \mathrm{N}$ to $20.75^{\circ} \mathrm{N}$ with $2.5^{\prime}$ resolution. They are shown in Fig. 12 by thin lines. For the ER these curves are so close to each other that can be easily interpolated by one profile shown in Fig. 12 by a solid thick line. The ridge width is defined with an accuracy of $\pm 5 \mathrm{~km}$ (see Fig. 12), so a dependence of the amplitudes on the ridge width $l$ should be used to estimate the range of generated amplitudes. In general, functions $a_{j}(l)$ reveal the resonance properties of the generation mechanism, i.e. strong dependence of the amplitude of generated waves on the relation between the wavelength of the generated mode $\lambda$ and the topography width $l$. In the considered case the ridge width $l$ is equal to $120 \pm 5 \mathrm{~km}$. This value is close to the width at which an absolute maximum of the function $a_{1}^{e}(l)$ is achieved (see Fig. 13). So, the linear
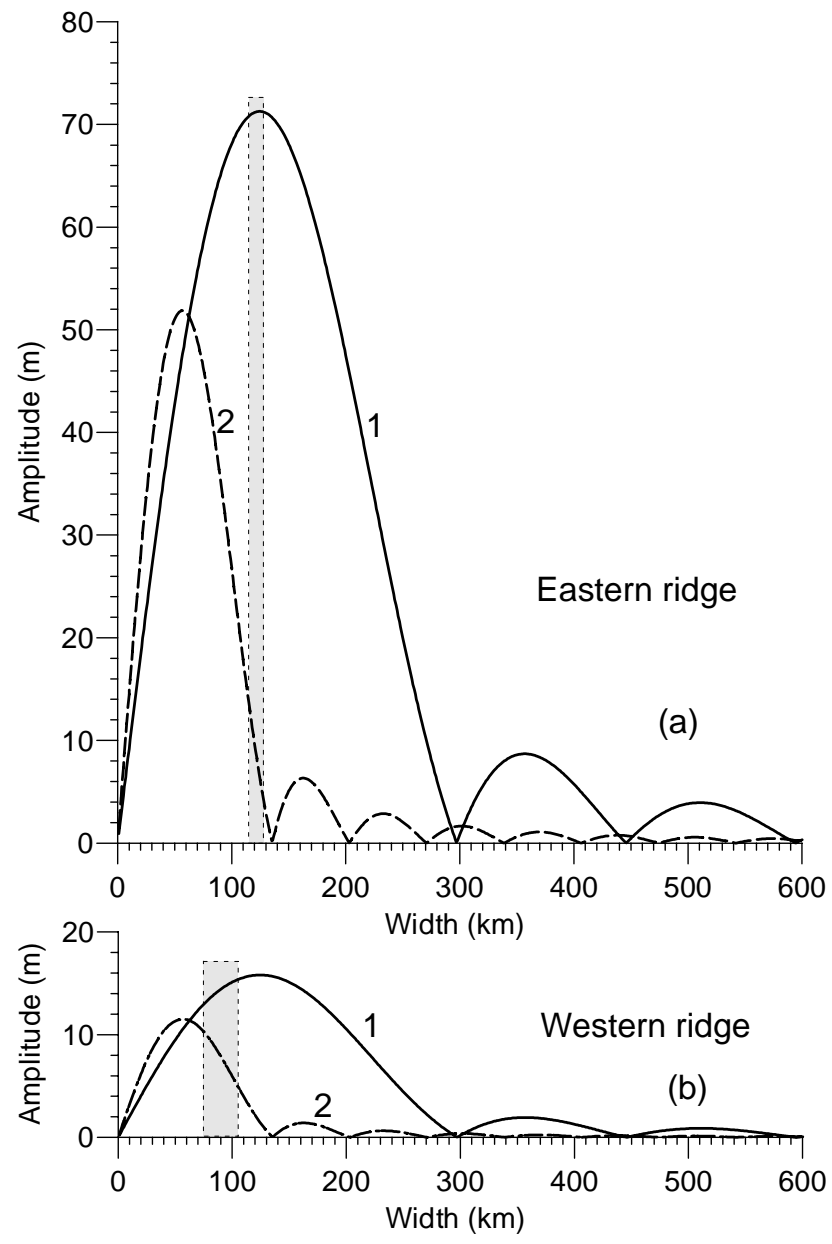

Fig. 13. Wave amplitudes of two first baroclinic modes (solid and dashed lines, respectively) generated by the eastern (a) and western (b) ridges, as predicted by linear theory. The uncertainty in definition of the ridge width is shown by grey boxes.

theory predicts quite a large amplitude of the first barolinic mode which in the considered case is equal to $71 \mathrm{~m} \pm 1 \mathrm{~m}$. At the same time the second-mode dependence $a_{2}^{e}(l)$ for the eastern ridge is close to its minimum: $a_{2}^{e}=8 \mathrm{~m} \pm 4 \mathrm{~m}$ for $l=120 \mathrm{~km} \pm 5 \mathrm{~km}$. Figure $11 \mathrm{~b}$ confirms that the second mode is negligibly small in comparison with the first one; only a weak second-mode signal can be detected beyond the ridge at the positions of vertical sections I and II.

The shape of the WR reveals variety in inclination of its western flank (see Fig. 12). The ridge topography here can be approximated by three different profiles, as it is shown by solid, dashed and dotted lines. In general, a better estimation for the width of the WR would be $l=90 \pm 15 \mathrm{~km}$. For this range the amplitudes of generated waves are found to be $14 \pm$ $2 \mathrm{~m}$ for the first mode, and $7 \pm 3 \mathrm{~m}$ for the second mode. The comparable amplitudes of the modes is the reason why the tidal beams are visible over the ridges, which is quite evident in Fig. 11c. 


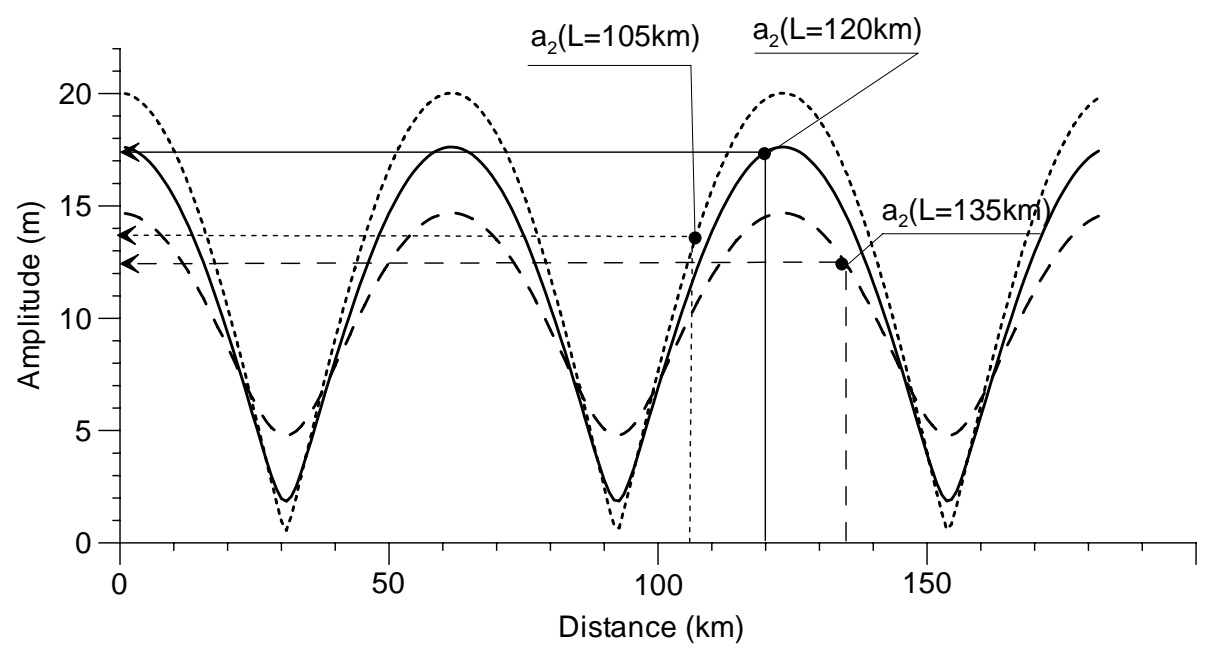

Fig. 14. Wave amplitudes of the second baroclinic mode generated by a two-sill topography as a function of the distance between the sills. Solid, dashed and dotted lines correspond to similar curves in Fig. 12.

In a linear case the resulting wave field is a sum of two solutions for the two-ridge topography. It is clear that two propagating waves can intensify or suppress each other depending on the phase shift between them, $\varphi_{j}^{e}-\varphi_{j}^{w}$. The final amplitude of the sum of two propagating second-mode waves generated over the western and eastern ridge can reach $20 \mathrm{~m}$ in a simple linear co-phase superposition when they are in phase, i.e. $\varphi_{2}^{e}=\varphi_{2}^{w}+2 \pi n, n=0, \pm 1, \pm 2, \ldots$ or can be close to zero when they are out of phase, $\varphi_{2}^{e}=\varphi_{2}^{w}+(2 n+1) \pi$, $n=0, \pm 1, \pm 2, \ldots$. An actual value of the phase shift $\varphi_{2}^{e}-\varphi_{2}^{w}$ between two waves generated by the ER and WR depends on the distance between them. The amplitude of the resulting wave is presented in Fig. 14 as a function of the distance between the ridges. Figure 12 suggests that the distance is in the range between 97.5 and $112.5 \mathrm{~km}$ and the resulting solution for the amplitude does not show any substantial amplification.

Note however that the decelerating effect of the variable topography of the WR on the propagating waves across it was missed in the proposed theory. This spatial lag is clearly seen from the comparison analysis of two temperature fields calculated for the full topography and without the western ridge (Fig. 11a and b). The estimation gives a value of 15 $\mathrm{km}$. The spatial lag together with the relative position of the two ridges $(L=105,120,135 \mathrm{~km})$ specify the range of possible summary amplitude of the second mode $a_{2}$ as shown in Fig. 14 by solid, dashed and dotted curves (the style of lines in Figs. 12 and 14 coincides).

It is clear that the wave amplification scenario is realized in the considered case: the two-ridge topography is in favor of a second mode generation. The resulting amplitude is close to its absolute possible maximum (its range is between $14.5 \mathrm{~m}$ and $17.5 \mathrm{~m}$ ). In reality the total amplitude can be even larger due to a nonlinear superposition of the interacting waves fields (Stashchuk and Vlasenko, 2005).

\section{Sensitivity runs}

Several additional numerical experiments were conducted to study the sensitivity of the model to various input parameters: external forcing, stratification and tidal frequency. The results are presented in Fig. 15 (a zonal section $20^{\circ} 46^{\prime} \mathrm{N}$ shown by line (b) in Fig. 3 is used for the analysis). Figure 15a illustrates the weakening of the baroclinic tidal signal with the decrease of the barotropic tidal forcing: $25 \%$ reduction of the velocity of the barotropic tidal flow slightly decreases the amplitude of the generated solitary internal waves, although the spatial structure and the modal content remain basically the same (compare solid and dashed lines in Fig. 15a). The four-fold reduction of the barotropic tidal forcing results in a dramatic weakening of the baroclinic tidal signal, especially its high-mode component. Qualitative analysis of the temperature sections in Fig. 15a shows that the higher baroclinic modes are almost invisible in the far-field.

Numerical experiments aimed to identify the difference between baroclinic wave fields generated under conditions of summer and winter stratification do not reveal any substantial discrepancy. Comparison of the two fields shown in Fig. $15 \mathrm{~b}$ shows quite similar spatial structures, including the number of generated waves, their amplitudes, and the presence of second-mode solitary waves with packets of shortscale waves developing on them. This similarity can be explained in terms of quite a weak inter-seasonal variability in water stratification. The latter remains almost unchanged over the year in the whole column except of the upper $250 \mathrm{~m}$ layer where the seasonal winter weakening of stratification is caused by the reduction of the surface temperature from 29 to $25^{\circ} \mathrm{C}$, which does not produce any radical changes (the buoyancy frequency maximum decreases from 0.015 to $\left.0.011 \mathrm{~s}^{-1}\right)$. 


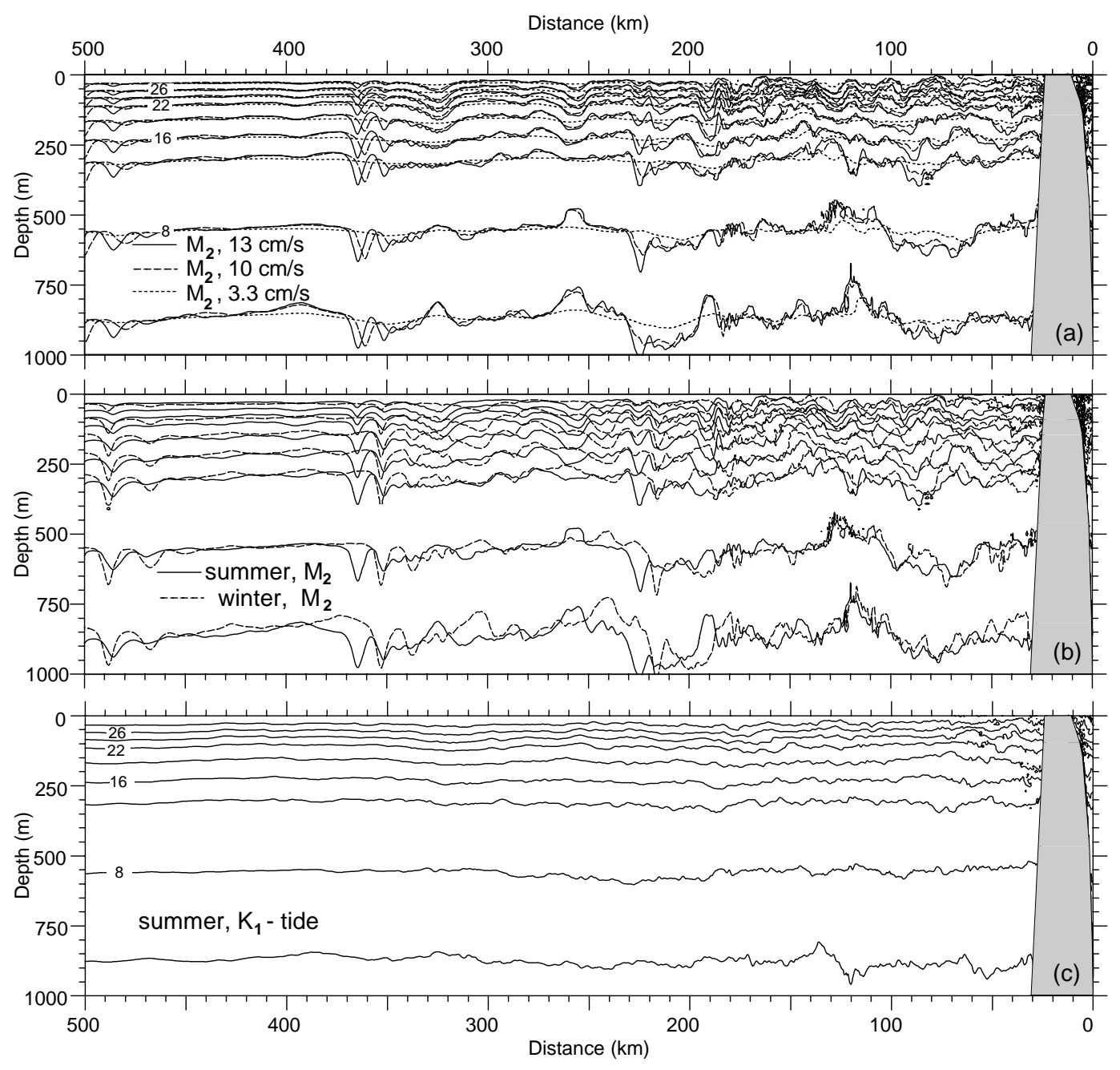

Fig. 15. Model-predicted temperature fields in the cross-section (b) shown in Fig. 3 after five tidal cycles calculated for (a) three tidal currents of different amplitude, (b) summer and winter stratification, and (c) $\mathrm{K}_{1}$ tidal harmonic.

The last numerical experiment, presented in Fig. 15c shows that $\mathrm{K}_{1}$ tidal harmonics (even being principal for the barotropic tide), is not able to generate a strong baroclinic tidal signal at the latitude $21^{\circ} \mathrm{N}$. The similar value of tidal forcing as in the reference experiment was used for the analysis. The temperature section shows that the diurnal baroclinic tidal field is fairly weak. The wave forms are randomly distributed and do not reveal any well-organized, and most importantly, quite intensive wave structures carrying energy from the ridges.

\section{Summary and conclusions}

We present here results of three-dimensional modelling of baroclinic tides in the area west of the Luzon Strait. SAR images (Zhao et al., 2004; Du et al., 2008) revealed two types of internal wave packets in the vicinity of the LS (to the east of $118^{\circ} \mathrm{E}$ ): single ISWs with horizontal scale of about $2.5 \mathrm{~km}$ and multi-wave packets of short waves with wavelengths less than $1 \mathrm{~km}$. This study was motivated by the intention to realize the conditions of their generation, their spatial structure, and the details of evolution.

The main focus of the modelling efforts was on an accurate reproduction of the mechanism of tidal energy conversion in the area of the Luzon Strait dominated by two ridges. These ridges are quite steep in terms of inclination of the bottom $\gamma$ and characteristic lines $\alpha$, which assumes generation of higher baroclinic modes, as predicted by linear theory. In the conditions when the nonlinearity is strong enough to contribute to the wave process, which is the case of the Luzon Strait, a multi-modal baroclinic bore with counterphase displacement of isopycnals/isotherms shown in Fig. 7a and $b$ is generated instead of a "traditional" co-phase firstmode baroclinic bore of depression/elevation usually developed in the course of nonlinear steepening of a propagating baroclinic tidal wave. The successive evolution of the "traditional" bore normally leads to a quick disintegration into 
a packet of rank-ordered ISWs according to the well studied "classical" mechanism of nonhydrostatic dispersion.

The process of evolution of a multi-modal baroclinic bore appears to be different. Several types of baroclinic wave forms can be generated in the course of its disintegration. A leading wave (or group of waves) with spatial structure peculiar to a first-mode ISW of depression is obviously generated at the leading edge of such a bore. This group of waves has the greatest phase speed, and therefore they detach fairly quickly from the wave tail and propagate independently as a packet of solitary waves of depression. The process of their separation and further independent propagation is illustrated in Fig. 7.

The remaining wave tail (see Fig. 8) possesses the characteristics of a packet of first-mode short-scale waves propagating on the background of a second-mode ISW whose vertical structure is shown in Fig. 6. Scrutiny of the spatial and temporal characteristics of the wave tail, i.e. the carrier second-mode ISW and the packet of short waves, was conducted on the basis of the model output and using the solution of the Taylor-Goldstein eigen-value problem. This analysis confirms that these short-scale waves are basically concentrated in the surface $500 \mathrm{~m}$ layer and attenuate exponentially below this depth (see Figs. 8 and 10). It is important that the short-scale wave packet and second-mode ISW are coupled: they propagate together with equal phase speed. In fact, the short waves riding the second-mode ISW exist only thanks to a specific vertical profile of the horizontal velocity produced by this mode as shown in Fig. 9. The solution of eigen-value problem (Eqs. 1-2) shows that the phase speed of these waves and their vertical structure are in a very good agreement with the numerically obtained short-scale waves (see Fig. 10). Being all the time behind the leading firstmode ISW and propagating together for a long distance, this coupled system produces quite a remarkable signal at the free surface (see Fig. 3, fragment $C$ ) which can erroneously be treated in analysis of remote sensing images as ordinary ISWs of depression, although they have a completely different structure.

The situation when solitary waves of mode greater than one can develop oscillatory wave tail of secondary short waves moving in resonance with them was discussed two decades ago by Akylas and Grimshaw (1992). Some numerical confirmations of this effect, i.e. the existence of solitary waves with secondary wave ripples were published (Hunter and Vanden-Broeck, 1983; Beale, 1991; Sun, 1991). In short, the physical mechanism of excitement of short-scale periodical waves is as follows. The first-mode solitary waves are supercritical, i.e. their speed is always higher than the linear long-wave-speed, and no wave coupling and resonance excitement of periodic internal waves can exist. At the same time the higher modes propagate slower, and there is a chance for periodical waves to be coupled with them with absorption of energy and growth in amplitude, Akylas and Grimshaw (1992).
An interesting result was obtained in a series of experiments aimed to identify the role of the eastern and western ridges in the generation process. A method of "truncated" topography was used, i.e. the numerical runs were conducted with ad-hoc bottom profile containing only one ridge in order to calculate their specific contribution to the resulting wave field. It was found that without the western ridge its eastern counterpart generates in the far-field a progressive first-mode tidal wave, which disintegrates into a packet of ISWs far beyond the LS. The higher modes are much weaker here and do not contribute much to the resulting wave field (see Fig. 11b). This is quite an unexpected result because the eastern ridge is steep enough to generate higher modes. An explanation was found in terms of a resonance theory of internal wave generation. The analytical solution obtained in the framework of a linear theory shows that the averaged width of the eastern ridge is close to the value when generation of the second mode is inhibited (Fig. 13a). At the same time the analytical solution predicts comparable amplitudes of the first and second modes generated by the western ridge (Fig. 13b). Thus according to the linear theory the western ridge, being deeper, mostly narrower and steeper than the eastern ridge, should generate higher baroclinic modes, which is really observed in the model output (see Fig. 11c). The wave signals produced by the eastern and western ridges interfere in the far-field, and their nonlinear superposition is substantially stronger than two separate signals (compare Fig. 11a, b and c).

Acknowledgements. This work was financially supported by National 863 High-tech Program (No. 2008AA09A402) of the Ministry of Science and Technology of China.

Edited by: K. Helfrich

Reviewed by: two anonymous referees

\section{References}

Akylas, T. R. and Grimshaw, R. H. J.: Solitary internal waves with oscillatory tails, J. Fluid Mech., 242, 279-298, 1992.

Beale, J. T.: Exact solitary water waves with capillary ripples at infinity, Commun. Pure Appl. Maths, 44, 211-257, 1991.

Buijsman, M. C., Kanarska, Y., and McWilliams, J. C.: On the generation and evolution of nonlinear internal waves in the South China Sea, J. Geophys. Res., 115, C02012, doi:10.1029/2009JC005275, 2010.

Duda, T. F., Lynch, J. F., and Irish, J. D.: Internal tide and nonlinear internal wave behavior at the continental slope in the northern South China Sea, IEEE J. Oceanic Eng., 29, 1-27, 2004.

Du, T., Tseng, Y.-H., and Yan, X.-H.: Impacts of tidal currents and Kuroshio intrusion on the generation of nonlinear internal waves in Luzon Strait, J. Geophys. Res., 113, C08015, doi:10.1029/2007JC004294, 2008.

Ebbesmeyer, C. C., Coomes, C. A., and Hamilton, R. C.: New observation on internal wave (soliton) in the South China Sea using acoustic Doppler current profiler, in: Marine Tech. Soc. 91 Proc., New Orleans, 165-175, 1991. 
Egbert, G. D. and Erofeeva, S. Y.: Efficient inverse modeling of barotropic ocean tides, J. Atmos. Oceanic Technol., 19(2), 183204, 2002.

Gerkema, T.: A unified model for the generation and fission of internal tides in a rotating ocean, J. Marine Res., 54, 421-450, 1996.

Helfrich, K. and Grimshaw, R.: Nonlinear disintegration of the internal tide, J. Phys. Oceanogr., 38, 686-701, 2008.

Hsu, M. K., Liu, A. K., and Liu, C.: A study of internal waves in the China Seas and Yellow Sea using SAR, Cont. Shelf Res., 20, 389-410, 2000.

Hunter, J. K. and Vanden-Broeck, J.-M.: Solitary and periodic gravity-capillary waves of finite amplitude, J. Fluid Mech., 134, 205-219, 1983.

Jan, S., Chern, C.-S., Wang, J., and Chao, S.-Y.: Generation of diurnal $\mathrm{K}_{1}$ internal tide in the Luzon Strait and its influence on the surface tide in the South China Sea, 112, C06019, doi:1029/2006JC004003, 2007.

Leith, C. E.: Stochastic models of chaotic systems, Physica D, 98, 481-491, 1996.

Marshall, J., Adcroft, A., Hill, C., Perelman, L., and Heisey, C.: A finite-volume, incompressible Navier-Stokes model for studies of the ocean on parallel computers, J. Geophys. Res., 102, 57335752, 1997.

Pacanowski, R. C. and Philander, S. G. H.: Parametrization of vertical mixing in numerical models of Tropical Oceans, J. Phys. Oceanogr., 11, 1443-1451, 1981.

Ramp, S. R., Tang, T. Y., and Duda, T. F.: Internal solitons in the northeastern South China Sea. Part I: Sources and deep water propagation, IEEE J. Oceanic Eng., 29, 1157-1181, 2004.

Shaw, P.-T., Ko, D. S., and Chao, S.-Y.: Internal solitary waves induced by flow over a ridge: with applications to the northern South China Sea, J. Geophys. Res., 114, C02019, doi:10.1029/2008JC005007, 2009.
Stashchuk, N. and Vlasenko, V.: Topographic generation of internal waves by nonlinear superposition of tidal harmonics, Deep-Sea Res. I, 52, 605-620, 2005.

Sun, S. M.: Existence of a generalized solitary wave solution for water with positive Bond number less than 1/3, J. Math. Anal. Appl., 156, 471-504, 1991.

Vlasenko, V., Stashchuk, N., and Hutter, K.: Baroclinic tides: theoretical modeling and observational evidence, Cambridge University Press, 2005.

Warn-Varnas, A., Hawkins, J., Lamb, K. G., Piacsek, S., ChinBing, S., King, D., and Burgos, G.: Solitary wave generation dynamics at Luzon Strait, Ocean Modell., 31, 9-27, doi:10.1016/j.ocemod.2009.08.002, 2010.

Yang, Y.-J., Tand, T. Y., Chang, M. H., Liu, A. K., Hsu, M.-K., and Ramp, S. R.: Solitons northeast of Tung-sha Island during the ASIAEX Pilot Studies, IEEE J. Oceanic Eng., 29, 1182-1199, 2004.

Zhao, Z., Klemas, V., Zheng, Q., and Yan, X.-H.: Remote sensing evidence for baroclinic tide origin of internal solitary waves in the northeastern South China Sea, Geophys. Res. Lett., 31, L06302, doi:10.1029/2003GL019077, 2004.

Zhao, Z. and Alford, M. H.: Source and propagation of internal solitary waves in the northeastern South China Sea, J. Geophys. Res., 111, C11012, doi:10.1029/2006JC003644, 2006.

Zheng, Q., Susanto, R. D., Ho, C.-R., Song, Y. T., and Xu, Q.: Statistical and dynamical analysis of generation mechanisms of solitary internal waves in the northern South China Sea, J. Geophys. Res., 112, C03021, doi:10.1029/2006JC003551, 2007.

Zu, T., Gan., J., and Erofeeva, S.: Numerical study of the tide and tidal dynamics in the South China Sea, Deep-Sea Res. I, 55, 137154, 2008.

World Ocean Atlas: http://www.nodc.noaa.gov/cgi-bin/OC5/ WOA05/woa05.pl, 2005. 